\title{
Major hepatectomy for hepatoma invading the main portal vein
}

\author{
R.C. Siriwardana, MBBS MS MRCS ${ }^{1}$, C.A.H. Liyanage, MBBS MS MRCS M Phil ${ }^{2}$, B.K.S. \\ Bulathsinhala, $\mathrm{MBBS}^{3}$ \\ 1 Consultant Gastrointestinal Surgeon, North Colombo Teaching Hospital, Ragama, Sri lanka. \\ 2 Senior Lecturer, Department of Surgery, University of Kelaniya, Medical College, Ragama, Sri Lanka. \\ 3 Surgical Registrar, Professorial Surgical Unit, North Colombo Teaching Hospital, Ragama, Sri Lanka.
}

Key words: Resection; Hepatocellular Carcinoma.

Resection or liver transplantation offers the only chance for cure in hepatocellular carcinoma (HCC). Portal vein tumour invasion is seen in $15-20 \%$ of the cases and carries a poor prognosis [1]. Most centres consider it a contraindication for aggressive treatment.

A 68 year old patient was found to have a $15 \mathrm{~cm}$ HCC in the right lobe of liver. The tumour was invading the right portal vein, main portal vein and extending to the left branch (Figure 1) (RPV). The liver was non-cirrhotic. Positron Emission Tomogram (PET) scan confirmed that there was no extra-hepatc disease. The left lobe measured $650 \mathrm{ml}$ and was adequate for surgical resection. Considering his excellent general condition and impending portal vein obstruction surgical resection was offered.

The anterior approach was used for the hepatectomy. Hilum was dissected and the portal vein was exposed after dividing the right hepatic artery and the right branch of bile duct. Proximal control of the portal vein was gained first, just above its origin. After dividing left caudate branches the left portal vein was controlled just before it entered the hilar plate (Figure 1).After clamping the main and left portal veins, right branch was opened longitudinally. The tumour thrombus was removed from the left and main portal veins. RPV was divided. Left portal vein clamp was removed to allow back bleeding. Portal vein was closed with 5/0 polypropylene. Transection was continued with cavitron ultrasonic suction aspirator (CUSA) and diathermy. The patient made an uneventful recovery. He is free of recurrence three months after surgery.

\section{References}

\section{Journal Articles}

1. Aldrighetti L, Pulitanò C, Catena M, Arru M, Guzzetti E, Halliday J, et al. Liver resection with portal vein thrombectomy for hepatocellular carcinoma with vascular invasion. Annals of Surgical Oncology. 2009; 16(5): 1254.

Doi: 10.1245/s10434-009-0383

PMID:19277788

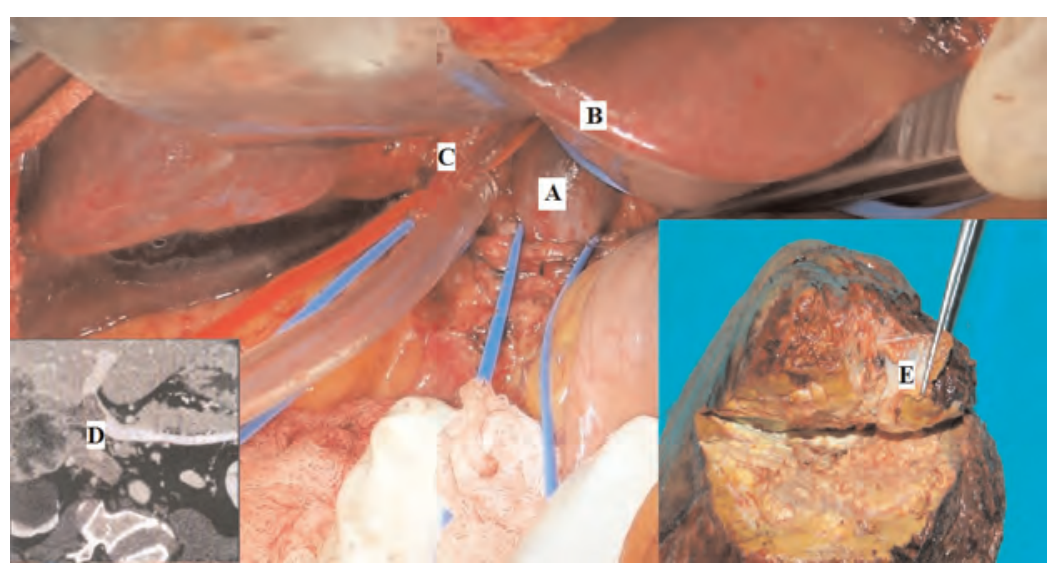

Correspondence: R.C. Siriwardana Consultant Gastrointestinal Surgeon, North Colombo Teaching Hospital, Ragama, Sri Lanka

E-mail: rohansiriwardana@yahoo.com
Figure 1. A- Main Portal vein, B- Left Portal Vein, CRight Portal Vein, D- Computed Tomogram showing Tumour invading the portal vein, E- Tumour in Main Portal Vein. 